\title{
LAS GENEALOGÍAS DE JESÚS
}

\section{Introducción}

En el transcurso de la Historia, tanto cristianos como judíos y paganos discutieron exacerbadamente sobre las genealogías de Jesús, que se encuentran en Mt 1,1-17 y Lc 3,23-38 (cf. las listas respectivas en los ítems 2.1 y 2.2 de este artículo). Esta discusión se debió al hecho de que ellas poseen entre sí enormes diferencias, a saber:

a) Mateo sigue un orden en línea descendente, con cuarenta y un nombres, y Lucas en línea ascendente, con setenta y siete nombres, más un excedente, o sea, Dios (cf. ítem 2.2 de este artículo); ${ }^{1}$

b) Mateo es más limitado, porque pretende solamente demostrar que Jesús es judío (por Abraham) y es descendiente de David, mientras Lucas añade a eso sus naturalezas humana (por Adán) y divina (por referir a Dios);

c) Mateo demuestra que Jesús tiene condición regia, relacionando los reyes de Judá a partir de Salomón, mientras que Lucas excluye esta condición, porque en su lista hay un desvío hacia otros descendientes de David que no fueron reyes, es decir, Natán (cf. 2 Sam 5,14 y 1 Cr 3,5 ) y sus sucesores desconocidos;

d) Lucas añade a la lista genealógica veintiún nombres anteriores a Abraham;

${ }^{1}$ En general las listas genealógicas bíblicas son en orden descendente. Pero hay excepciones, como las de $1 \mathrm{Cr} 6,18-32$ y, en la LXX, Jdt 8,1. 
e) de David a Salatiel, Mateo relaciona dieciséis nombres y, de Zorobabel hasta Jesús, doce nombres. Lucas menciona, respectivamente, veintidós y veintiún nombres;

f) mientras Mateo hace supresiones y no repite nombres, Lucas aparentemente no realiza exclusiones y repite sistemáticamente varios nombres;

g) de Abraham hasta David, las listas se asemejan; solamente se vuelven a encontrar en Salatiel, Zorobabel, José y Jesús;

h) Mateo coloca Salatiel y Zorobabel en la rama de los reyes y Lucas no;

i) Lucas escribe algunos nombres de forma diferente que Mateo: Arní en vez de Aram; Salá en vez de Salmón; y Booz en vez de Boez;

j) Mateo, al contrario de Lucas, cita los nombres de algunas madres: Tamar, Rahab, Rut, la mujer de Urías (Betsabé) y María; ${ }^{2}$

k) de la misma forma, Mateo cita hermanos: los hermanos de Judá, y Zera, hermano de Fares; ${ }^{3}$ y

2 Probablemente esto sucede para que existiera la oportunidad de justificar la presencia de María (cf. Mt 1,16), lo que era necesario hacer debido a la concepción virginal (cf. Mt 1,18). Algunos estudiosos dicen que las mujeres mencionadas por Mateo son tipos de María, en la medida en que el poder de Dios se manifestó a ellas (cf. J. Jeremias, Jerusalem in the Time of Jesus, Fortress - Minneapolis - 1975, 293). Este parecer se confirmaría con la situación bíblica irregular de las mujeres citadas por Mateo, la cual puede ser equiparada a la concepción extraconyugal de la madre de Jesús (para Tamar, cf. Gn 38,6-30; para Rahab, cf. Jos 2; 6,17.25, a pesar de que en Mt 1,5 parece haber ocurrido una atribución ficticia y forzada de maternidad, porque no hay mención del nombre Rahab en las listas genealógicas de $1 \mathrm{Cr} 2,12$ y Rut 4,21; para Rut, cf. Rut 2,8-13; 3,7-18; 4,13; y para Betsabé, cf. 2 Sam 11). Otros, sin embargo, prefieren ver en las mujeres una intención universalista de Mateo con respecto a los destinatarios de su evangelio, considerando que ellas no son judías (Tamar a veces fue vista como prosélita; Rut era moabita; Rahab era una residente de Jericó; y Betsabé posiblemente era una hitita, como su marido Urías). Para las varias hipótesis sobre este tema, cf. M. D. JoHnson, The Purpose of the Biblical Genealogies with Special Reference to the Setting of the Genealogies of Jesus, NTSMS 8, Cambridge University - Cambridge - 1969, 152-179; R. E. BROWN, The Birth of the Messiah, Doubleday - Garden City/New York - 1977, 71-74; C. I. BLOMBERG, "The Liberation of Illegitimacy: Women and Rulers in Matthew 1-2", BTB 21 (1991) 145-150; R. BAUCKHAM, "Tamar's Ancestry and Rahab's Marriage: Two Problems in the Matthean Genealogy", NovT 37 (1995) 313-329; J. Nolland, "Genealogical Annotation in Matt. 24:34: A Literary Critical Perspective", JETS 38 (1995) 369-385; W. J. C. WEREN, "The Five Women in Matthew's Genealogy", CBQ 59 (1997) 288-305; W. D. DAVIES - D. C. AlLISON, Matthew 1-7, ICC, T \& T Clark - London - 2004, 170-172; y U. LUZ, Matthew 17, Hermeneia, Augsburg Fortress - Minneapolis - 2007, 83-85. 
1) Mateo afirma que Jeconías es padre de Salatiel, Zorobabel es padre de Abiud y Jacob es padre de José, mientras Lucas asevera que Nerí es padre de Salatiel, Zorobabel es padre de Resá y Elí es padre de José.

Por su lado, los cristianos intentaron justificar algunas de estas diferencias por medio de tesis de armonización, debido a la necesidad teológica de que haya solamente una genealogía. También sugirieron hipótesis de inclusión de María en una de las dos listas genealógicas, porque Jesús tenía que ser descendiente carnal de David por medio de su concepción virginal.

Sin embargo, las propuestas se basan en meras suposiciones y en artificios forzados. Esta base conjetural y artificiosa atribuye a la cuestión una complicación no convincente e inaceptable desde el punto de vista de un estudio crítico.

Por otro lado, a veces esta complicación ha sido adoptada hasta en estudios críticos, prefiriéndose formular proposiciones incongruentes que pecan por la falta de un análisis adecuado y minucioso. Esta tendencia ha llevado a muchos engaños que empeoran aún más la cuestión. Por este motivo, es necesario rever las tesis que involucran las genealogías de Jesús.

El presente artículo se propone revisarlas y emprender un análisis minucioso de la estructura de las listas y de los nombres que las componen. Para eso serán presentados nuevos detalles y observaciones que pueden conducir a soluciones más lógicas, apropiadas y esclarecedoras.

\section{Las tesis sobre las listas de Mt 1,1-17 y Lc 3,23-38}

\subsection{Retrospectiva de las tesis}

A lo largo del tiempo, la controversia de las listas genealógicas se centralizó más en la cuestión de los nombres diferentes de los padres (cf. letra 1 arriba). La solución sugerida para este problema fue la armo-

${ }^{3}$ Tal vez éstos estén de paso en la lista porque remiten, respectivamente, a los doce hijos de Jacob y al hecho de que Fares y Zera eran mellizos (Gn 38,27-30). Para esta cuestión, cf. DAVIES - AlLISON, Matthew 1-7, 169-170. 
nización entre ellas. Julio Africano (ca. 240 d. C.) fue el primero en armonizarlas por medio del levirato. ${ }^{4}$

El levirato era una regla judía que determinaba el casamiento del cuñado con una cuñada cuando ésta quedase viuda (cf. Dt 25,5-10; Gn 38,8). Africano, basándose en esa ley, supone que Jacob y Elí eran hermanos por parte de madre, de la siguiente forma: Matán, descendiente de Salomón, engendró a Jacob; habiendo muerto aquél, Melquí (en realidad, Matat, de acuerdo con Lucas; cf. ítem 2.2 de este artículo), ${ }^{5}$ descendiente de Natán, se casó con la viuda de Matán y engendró a Elí; muerto Elí, Jacob desposó su mujer y engendró a José; sin embargo, legalmente José era considerado también hijo de Elí; de esta forma, según propone Africano, Mateo nos da la genealogía de los ascendientes naturales de Jesús, mientras que Lucas nos ofrece la de sus ascendientes legales.

Por otro lado, Eusebio consideró que María debería ser de la misma tribu que José, porque la ley mosaica no permitía el casamiento con miembro de otra tribu (cf. Nm 36,8-9). ${ }^{6}$ Los Padres de la Iglesia, más específicamente, fueron unánimes en aseverar que María era descendiente de David, debido a la necesidad teológica de sostener el origen carnal davídico de Jesús, de acuerdo con la interpretación literal de Rom $1,3^{7}$

Otros añaden que María también sería hija de Elí y, por tanto, media hermana de José. Así, la lista de Lucas nos traería los ancestros de María, y, la de Mateo, los de José. ${ }^{8}$ En el intento de confirmar esta

${ }^{4}$ Cf. Julio Africano, Carta a Aristides, en Eusebio, Hist. Eccl. 1.7.1-17.

${ }^{5}$ Africano parece ignorar el nombre correcto, o sea, Matat. Tal vez, él tuviera en su poder una lista incompleta. Este defecto ocurre en otros manuscritos, como B N U (setenta y seis nombres) y sy. ${ }^{\text {sin }}$ (setenta y tres nombres). IRENEO, en Adv. Haer. 3.22.3, nos habla de setenta y dos nombres. Existen varios manuscritos latinos que también presentan setenta y dos hombres. Por otro lado, algunos piensan que la lista de LC 3,23-38 tenía originariamente menos nombres, en vez de setenta y siete, considerando que Africano, inclusive, desconoce Matatá y Leví, así como en otros manuscritos faltan Aminadab y Menná (respectivamente, B y A).

${ }^{6}$ Cf. Eusebio, Hist. Eccl. 1.7.17.

7 Por ejemplo: Justino, Dial. 100; IgnACIO, Eph. 18; IRENEO, Adv. Haer. 3.21.5; Orígenes, Contra Celsum 2.32 (PG 2, 852); EPIFANIO, Haer. 51.11; Agustín, Contra Faustum 1.2 ( $P L$ 42, 471-472), donde él interpreta Rom 1,3 en el sentido biológico.

8 Según F. X. PAtrizzI, De Evangeliis, Freiburg in Breisgau - 1853, II, 84-91, esta tesis habría sido formulada por primera vez por Anio de Viterbo (ca. 1490), en su comentario sobre la obra Breviarium de Temporibus, de Filón. 
hipótesis, están los que mencionan el pasaje del tratado rabínico Hăgigāah $77 \mathrm{~d}$, del Talmud de Jerusalén, donde se encuentra un personaje llamado "María, hija de Elí"."

Otra explicación sería la adopción de José por parte de Elí, padre de María, la cual no habría tenido hermanos (cf. Esd 2,61; Ne 7,63; $1 \mathrm{Cr}$ 2,21-22.34-35; Nm 27,3-11). ${ }^{10}$

Recientemente surgió una nueva tesis, basada en genealogías judías del período del Segundo Templo, en las cuales eran comunes los nombres José y María. Por causa de esto, esta tesis defiende que existirían cuatro fuentes genealógicas diferentes e independientes formadoras de las genealogías de Mateo y Lucas, y que habría habido una yuxtaposición incorrecta entre ellas, de manera que María, madre de Jesús, habría tenido tanto un padre como un marido llamados José, siendo el primero hijo de Elí, y, el segundo, hijo de Jacob. ${ }^{11}$

Con respecto a los nombres del padre de Salatiel (Jeconías-Nerí) y del hijo de Zorobabel (Abiud-Resá), existen problemas no solamente internos sino también externos a las listas. En $1 \mathrm{Cr} 3,17$, en concordancia con Mateo, Jeconías es padre de Salatiel, y no Nerí: éste es un desconocido. Sin embargo, tanto Mateo como Lucas difieren de $1 \mathrm{Cr}$ 3,19-20: los nombres Abiud y Resá no aparecen entre los hijos de

${ }^{9}$ La cita de yHag 77d fue hecha por primera vez por JOHN LIGHTFOOT, en Horae Hebraicae et Talmudicae in quattuor Evangelistas, editada en Leipzig por J. B. Carpzov, en 1679. Existe una repetición sintetizada del mismo relato en ySan 23c. Por otro lado, la tesis de que el padre de María se llama Elí choca con la tradición cristiana del siglo II d. C., expresada en el Proto Evangelio de Santiago, en el cual el padre de María se llama Joaquín, no Elí. CORNELIO À LAPIDE, The Great Commentary, Luke 3:23 (ed. John Hodges et al.), London - $1890^{4}$, intenta conciliar a Elí con esta tradición, considerando que este nombre puede ser una abreviación de Eliaquim, el cual se trata de una alternativa para el nombre Joaquín (cf. 2 Re 23,34 y 2 Cr 36,4). En contrapartida, JUAN DAMASCENO, Fide Orthodoxa 4.14, afirma que Elí fue primo de José, y PATRIZzI, De Evangeliis, 98, partiendo de un análisis del texto de Juan Damasceno, entiende que José fue tío de María.

${ }^{10}$ De acuerdo con la propuesta de V. U. HolzMEISTER, en "Ein Erklärungsversuch der Lk-Genealogie (3,23-28)", ZKT 47 (1923) 184-218; y de L. NolLE, en "Old Testament Laws of Inheritance When He Taught", NTS 5 (1958-59) 291-298. Pero Agustín, en De Consensu Evang. 2.4 (PL 34, 1072-1073), ya había formulado esta propuesta, a pesar de que más tarde acabó desistiendo de ella (cf. Retractat. 2.12, PL 32, 632-633). Recientemente, Y. LEVIN, "Jesus, Son of God and Son of David: The Adoption of Jesus into the Davidic Line", JSNT 28 (2006) 415-442, demostró que esta tesis puede ser descartada, ya que este sistema de adopción era desconocido por la Ley judía en la época del nacimiento de Jesús.

${ }^{11}$ Esta tesis fue formulada por B. SIVERTSEN, "New Testament Genealogies and the Families of Mary and Joseph", BTB 35 (2005) 43-50. 
Zorobabel. Las soluciones que se dieron para tales divergencias son las siguientes:

a) Melquí, por levirato, se casó con la viuda de Joaquín (este nombre no aparece en Mt 1,11-12; cf. ítem 2.1 y nota 23 de este artículo), padre de Jeconías, engendrando a Nerí. Por lo tanto, Jeconías sería hermano de Nerí por parte de madre. Muriendo Nerí, Jeconías se casó con su viuda, engendrando con ésta a Salatiel; ${ }^{12}$

b) no se ha dado explicación para que un tal Abiud sea hijo de Zorobabel, pero, para Resá, se ha pensado que este nombre debe ser entendido como un atributo de Zorobabel, el cual habría sido "príncipe" -en arameo rē'ša - de Judá, como era su antecesor Sesbasar (cf. Esd 1,8). De acuerdo con este pensamiento, Joanán debe ser identificado con Ananías, hijo de Zorobabel (cf. $1 \mathrm{Cr}$ 3,19.21), pues Joanán es una forma nominal alternativa para Ananías (ambos significan "Dios es misericordioso"). Así, el conjunto Joanán-Resá-Zorobabel debe ser interpretado de la siguiente manera: Ananías (hijo del) príncipe Zorobabel. ${ }^{13}$

\subsection{Objeciones a las tesis y soluciones para las genealogías de Jesús}

En realidad, todos estos intentos no son convincentes, teniendo en cuenta las siguientes objeciones:

a) no se puede admitir que los reyes relacionados por Mateo fuesen tan insignificantes al punto de que Lucas los desprecie. O sea, de un modo u otro, si los reyes eran verdaderos ascendientes de Jesús, Lucas jamás podría desviarse hacia Natán. Siendo así, no habría motivos, inclusive, para que Lucas despreciase a los descendientes de Salomón cercanos a José -Matán y Jacob-, ya que venían de una rama más importante;

12 De acuerdo con la sugerencia de A. PLUMmer, A Critical and Exegetical Commentary on the Gospel According to S. Luke, Scribner - New York - 1922, 104; e I. H. MARSHALL, The Gospel of Luke: A Commentary on the Greek Text, Paternoster - Exeter $-1978,163$.

${ }^{13}$ Cf. A. Hervey, The Genealogies of Our Lord and Saviour Jesus Christ, as Contained in the Gospels of St. Matthew and St. Luke, MacMillan / Hatchard - Cambridge / London - 1853, 111-121, fue quien formuló esta hipótesis. 
b) la hipótesis de Africano es muy seductora en sus argumentos, e incluso muy versátil en el detalle de que puede ser concebida no sólo partiendo del lado de Mateo sino también del lado de Lucas, de la siguiente manera: Matat, descendiente de Natán, engendró a Elí; después de su muerte, su mujer se casa con Matán, descendiente de Salomón; de esta unión nace Jacob; al morir Jacob, Elí se casa con su viuda, quien acaba engendrando a José, pero legalmente es Jacob el padre de José. En esta alternativa, Mateo ofrece el linaje legal y Lucas el natural. ${ }^{14}$ Obviamente, Africano debe haber optado por la alternativa de Mateo porque ese evangelio relaciona los reyes de Judá como ascendientes de Jesús, prefiriendo dar una calidad regia a este último. Sin embargo, la tesis de Africano posee un grave defecto: se restringe solamente a una parte de las generaciones, olvidándose de todo lo restante e ignorando el problema de la presencia de Salatiel y Zorobabel en ambas listas, lo cual resalta los siguientes hechos: contradictoriamente, la genealogía de Mateo está compuesta por reyes, de Salomón hasta Jeconías, mientras que la de Lucas no; y los nombres antes de Salatiel y después de Zorobabel son totalmente diferentes entre las listas;

c) la propuesta de levirato entre Melquí y Joaquín podría dar un acabado para la tesis de Africano. Sin embargo, ella es fácilmente disuelta en el momento en que percibimos que el hijo de Joaquín fue ignorado por Mateo (en realidad, el Jeconías de Mt 1,11-12 es Joaquín, padre de Jeconías; cf. ítem 2.1 y la parte final de la nota 23 de este artículo) y que el padre de Melquí y Joaquín tendría que ser obligatoriamente el mismo (en Mateo es Josías y en Lucas es Addí). Esta propuesta trae además otra complicación: el levirato entre Melquí y Salatiel origina que se vuelva a la lista de Lucas, considerándose que Abiud es desconocido. Este retorno a Lucas acaba invalidando completamente a Mateo a partir de Abiud; ${ }^{15}$

${ }^{14} \mathrm{Cf}$. Hervey, Genealogies, 13, defiende esta tesis alternativa, contraria a la de Africano, de que Mateo nos da la línea legal y Lucas la línea natural.

15 Se puede pensar que Mateo salteó los hijos de Zorobabel, saltando hasta Abiud, obrando también de esta forma entre algunos de los demás nombres subsiguientes, con la finalidad de reducir el número de ellos hasta 14. Pero, igualmente, los nombres que él relaciona nunca coinciden con los de Lucas, lo que en 
d) también es difícil aceptar la tesis de que Resá sea solamente un atributo en vez de un nombre, teniendo en cuenta que Resá tiene que ser obligatoriamente un antepasado para que se complete la cantidad exacta de veinte nombres en la primera parte del grupo de cuarenta nombres donde se encuentran los ascendientes desconocidos de Jesús (excluyéndose Zorobabel y Salatiel; cf. Lc 3,23-31, e ítem 2.2 de este artículo). Además, el nombre Jodá, que antecede Joanán, no aparece en la lista de los hijos de Ananías en 1 Cr 3,21. Este detalle nos lleva a la conclusión de que el Joanán que está en la genealogía de Lucas no es Ananías;

e) la hipótesis de que Mateo nos da los ancestros de José, y Lucas los de María, no tiene fundamento, porque los evangelistas no estaban preocupados por esta distinción: tanto uno como el otro deseaban explicitar las genealogías de José, y con bastante vehemencia. La insistencia en que es José el descendiente de David está bien expresada en Mt 1,20 y en Lc 1,27; 2,4. Sobre el origen de María ellos no declaran nada. Ese hecho tuvo una razón bien evidente: entre los judíos, una genealogía solamente podría ser formada por medio de los varones ancestrales del padre del descendiente en cuestión. Por eso, ambas genealogías, para que tuvieran credibilidad, no podrían basarse en ascendientes de la madre de Jesús; ${ }^{16}$

f) además, si es biológica y teológicamente necesario que María sea de la tribu de Judá y descendiente de David, entonces, en lugar de buscar explicaciones complicadas, como la del levirato, sería preferible imaginar hipotéticamente que María fuera descendiente por medio de uno de los demás hijos de David, aparte de Salomón y Natán (cf. 1 Cr 3,1-9); ${ }^{17}$

g) siendo evidente que los evangelistas tenían que explicitar solamente genealogías de José y que María podría tener origen en otro

algún momento debería suceder, aún más porque Lucas nos da un número de nombres más confiable.

${ }^{16}$ Las genealogías tendrían que ser hechas por el padre y los respectivos varones ancestrales, aunque el descendiente en cuestión fuese una mujer, como vemos en $1 \mathrm{Cr}$ 2,34-41 y Jdt 8,1.

${ }^{17}$ Sin embargo, se debe recordar que Lc 1,5.36 parece insinuar que María era de linaje sacerdotal, porque Isabel, una descendiente de Aarón, era su parienta. A pesar de eso, de acuerdo con J. A. FitzMYer, The Gospel According to Luke, Doubleday Garden City/New York - 1981, I, 357, existe mucha probabilidad de que tal insinuación sea creación de Lucas. 
hijo de David, las hipótesis de linajes legales y naturales, de adopción y de yuxtaposición genealógica incorrecta, quedan sin sentido, tratándose, en realidad, de tesis forzadas e ingeniosas, creadoras de suposiciones que sobrepasan el texto de los evangelios y la intención original de los evangelistas. Lo mismo se debe decir con respecto a la idea del levirato;

h) finalmente, Hăgigāh 77d no permite pensar que el personaje llamado María que de allí surge sea la madre de Jesús, porque dicho texto se refiere a la hija de Elí Bitsalim, vista en un sueño por un hombre santo judío. El personaje se presenta allí con una función meramente figurativa y, de acuerdo con el contexto, parece relacionarse con un hechizo. Por lo tanto, no existe ninguna posibilidad de identificación y confirmación. ${ }^{18}$

Ante todo esto, podemos concluir que todas las tesis mencionadas son inválidas. En realidad, sea desde un análisis por separado, sea por los intentos de armonización, las dos listas son incompatibles y, sin duda, Lucas no conocía la lista de Mateo, y tampoco éste la de Lucas. ${ }^{19}$

Debido a esta conclusión, es necesario formular otras tesis que sean más lógicas para explicar las genealogías de Jesús.

A continuación intentaremos emprender esta formulación, por medio de un análisis minucioso que tenga en consideración:

a) los sistemas empleados por los evangelistas para formar las listas;

b) la morfología y los significados de los nombres que las componen;

c) la comparación entre los nombres de las listas y otros nombres bíblicos; $\mathrm{y}$

d) las posibles relaciones históricas, bíblicas, teológicas, analógicas y tipológicas que las involucran.

${ }^{18}$ En el texto de $y H a g$ 77d se habla de "Miriam, la hija de Elí Bitsalim". Este último nombre puede ser traducido por "Elí Cebolla". En el pasaje, Miriam surge en el sueño de un judío santo, colgada por las mamilas de sus senos, en cuya oreja quedará fijado el barrote del portón de la Gehena hasta que venga Simeón Bar Shatah. Notablemente, el personaje es muy alegórico, representando alguna situación inmoral ocurrida en la época en que el texto fue escrito.

19 De acuerdo con F. D. BRUner, Matthew: A Commentary, The Christbook, Matthew 1-12, Eerdmans - Michigan - 2007, 15, la recomendación actual de la mayoría de los estudiosos es no intentar armonizar las genealogías. 


\section{Análisis de las genealogías de Jesús}

\subsection{La genealogía de Mt 1,1-17}

La genealogía de Mt 1,1-17 pretende demostrar, principalmente, que Jesucristo tiene una ascendencia regia, representada por David y sus reyes descendientes, reivindicándole el derecho de herencia al trono de David y la calidad de Mesías. Secundariamente, pretende afirmar además que Jesús era un legítimo judío, por medio de la figura de Abraham.

Además de estos objetivos explícitamente declarados en el primer versículo, el evangelista parece querer dar a su genealogía un aspecto de perfección, al observar en el versículo 17 que existen tres conjuntos de generaciones, formados, cada uno, por el número 14 , totalizando cuarenta y dos generaciones. Aparentemente, este acabado numérico dejaría traslucir que el linaje regio, la cronología y la historia judía fueron circunstancias determinadas por un plan divino oculto y perfecto. ${ }^{20}$

Veamos cómo se componen estos conjuntos, obedeciendo a la configuración de Mt 1,2-16 y transcribiendo en castellano de la manera que sea más cercana al original griego:

1. Abraham, Isaac, Jacob, Judá, Fares, Esrom, Aram, Aminadab, Naasón, Salmón, Boez, Jobed, Jesé, David.

2. David, Salomón, Roboam, Abiá, Asaf, Josafat, Joram, Ozías, Joatam, Ajaz, Ezequías, Manasés, Amós, Josías, Jeconías.

3. Jeconías, Salatiel, Zorobabel, Abiud, Eliaquim, Azor, Sadoc, Ajim, Eliud, Eleazar, Matán, Jacob, José, Jesús.

Notablemente, al contrario de lo que afirma el evangelista en el versículo 17, en realidad lo que existe en Mt 1,2-16 son catorce nombres

${ }^{20}$ Algunos estudiosos afirman que Mateo adoptó el número 14 porque pensaba en la gematría del nombre David, la cual resulta en 14 (cf. JeREMIAS, Jerusalem, 292). DAVIES - Allison, Matthew, 165, entienden que el nombre David es la clave para el patrón numérico de la lista de Mateo, porque aparece en el décimo cuarto lugar y porque posee tres consonantes, simbolizando los tres conjuntos o períodos históricos de catorce generaciones. Sin embargo, lo más lógico sería pensar que el patrón numérico de Mateo nació de la simple observación del evangelista de que el primer conjunto, de propósito iniciado y terminado por nombres importantes, o sea, Abraham y David, sumaba catorce nombres. Debido a este hecho, resolvió imponer este patrón a los demás conjuntos, aunque fuera de manera aparente y artificial, con la intención de dar la impresión de que hubo ciclos históricos perfectos. 
en el primer conjunto, catorce generaciones en el segundo (el cual, por lo tanto, posee quince nombres) y catorce nombres en el tercero. ${ }^{21}$ De esta forma, el único que contiene catorce generaciones es el segundo conjunto, siendo que los demás se componen de trece generaciones cada uno, totalizando cuarenta generaciones, en vez de cuarenta y dos. ${ }^{22}$

Además de esto, el evangelista omitió algunos reyes, que fueron Ocozías, Joás y Amasías, entre Joram y Ozías; y consideró intencionalmente al rey Joaquín como si fuera Jeconías, que fue su hijo y sucesor, haciendo que este "Joaquín-Jeconías" se transformase en padre de Salatiel. $^{23}$ También eliminó a Ananías, Mesulam, Secanías, Nearías y Elioenay, descendientes principales que ocurrieron después de Zorobabel.

21 Los conjuntos de generaciones podrían haber sido formados de la siguiente manera: a) de Téraj-Abraham (cf. Gn 11,27) hasta Jesé-David; b) de David-Salomón hasta Josías-Joaquín (cf. $1 \mathrm{Cr} 3,15-16)$; c) y de Joaquín-Jeconías hasta José-Jesús. El aparente engaño de Mt 1,17 se debe al deseo forzado de iniciar por Abraham (cf. Mt $1,1)$, de suprimir algunos nombres y de dividir la lista en tres fases históricas con números simbólicos idénticos.

22 El evangelista añadió la generación Josías-Jeconías al final del segundo conjunto (Mt 1,11). Debido a esta decisión, el segundo conjunto acabó siendo formado realmente por catorce generaciones. Para las varias hipótesis sobre este tema, cf. Donald A. HAGner, Matthew 1-13, WBC 33A, Word Books - Dallas - 1993, 5-6.

${ }^{23}$ La supresión de Ocozías, Joás y Amasías puede haber sido el resultado de una actitud purista del evangelista, considerando que esos reyes fueron pecadores (cf. $2 \mathrm{Cr}$ 22,3-4; 24,17-27; 25,14-16), o de un error (cf. J. JeremiAS, Jerusalem, 294). También hay quien piensa que la eliminación se dio porque aquellos reyes fueron asesinados. Sobre la supresión de Joaquín, se entiende que hubo un error a causa de la semejanza entre los nombres de Joaquín y Jeconías, también llamado Joaquín (cf. 2 Cr 36,9-10). Para esta interpretación, cf. J. MASSON, Jésus, Fils de David dans les Généalogies de Saint Matthieu et de Saint Luc, Téqui - Paris - 1982, 42-55; y A. VöGTLE, "Josias zeugte den Jechonias und seine Brüder (Mt 1,11)", Lex tua Veritas, FS H. Junker, Paulinus Trier - 1961, 307-313, y "Die Genealogie Mt 1,2-16 und die matthäische Kindheitsgeschichte", BZ.NF 8 (1964) 45-58, 239-262; 9 (1965) 32-49, reimpreso en Das Evangelium und die Evangelien, Patmos - Düsseldorf - 1971, 57-102. J. NolLAND, en "Jechoniah and His Brothers (Matthew 1:11)", BBR 7 (1997) 169-178, afirma que la expresión "Josías engendró a Jeconías y a sus hermanos" de Mt 1,11 -la cual sería errónea, ya que Josías, en realidad, fue abuelo de Jeconías, y éste tuvo sólo un hermano, como se ve en $1 \mathrm{Cr}$ 3,15-16- indica que Mateo entendía implícitamente en Jeconías a su padre Joaquín, para que pudiese evocar aspectos significativos del período de la conquista babilónica y comprender las dos generaciones finales de la monarquía de Judá. Sin embargo, a decir verdad, parece que Mateo quería simplemente que el rey Joaquín fuese confundido con Jeconías para que pudiese saltar directamente hacia Salatiel. De esta forma, desde el punto de vista de la intención del evangelista, el inicio del tercer conjunto sería "correcto", ya que Jeconías fue padre de Salatiel (cf. $1 \mathrm{Cr} 3,17)$, y la expresión "Josías engendró a Jeconías y a sus hermanos" no estaría equivocada, porque el "Joaquín-Jeconías" de Mateo realmente tuvo tres hermanos (cf. $1 \mathrm{Cr} 3,15)$. 
Encontramos estos nombres faltantes en 1 Cr 3,10-24. Así, Mt suprimió, por lo menos, nueve personajes, con la finalidad obvia de conformar su genealogía con el principio del número 14. Lo mismo se puede decir con respecto a los nueve nombres relacionados entre Zorobabel y José: como mínimo, tendría que haber en este interregno cerca de veinte generaciones, considerándose el espacio de casi seiscientos años. ${ }^{24}$

La fuente inicial del evangelista para componer su lista puede haber sido 1 Cr 1-3, con excepción de que prefirió seguir la tradición de Ag 1,1, Esd 3,2 y Ne 12,1 con respecto a Salatiel, considerando que $1 \mathrm{Cr}$ 3,19 afirma que el padre de Zorobabel era Pedaías, no Salatiel. ${ }^{25}$

Los nombres que se encuentran entre Zorobabel y José forman un conjunto de nueve antepasados desconocidos. A continuación analizaremos a cada uno de ellos.

Abiud es un nombre que se repite solamente en $1 \mathrm{Cr} 8,3$, remitiendo a uno de los nietos de Benjamín, hijo de su primogénito Bela. Como este personaje tiene descendencia en Jacob y ascendencia en el rey Saúl (cf. 1 Sam 9,1-2; 1 Cr 8,33; 9,39), y Benjamín es constantemente mencionado con Judá (cf. 2 Cr 15,2.8-9; 25,5; 31,1; Esd 4,1-2; 10,9), la presencia de este nombre en la lista puede estar indicando una vinculación de Jesús a la calidad regia de la tribu de Benjamín, compañera de Judá (cf. 1 Re 12,21; 2 Cr 11,10-12.22-23), y tal vez tenga que ver con el nombre José, que recuerda al hermano de Benjamín y al padre de Jesús.

Eliaquim remite al rey de Judá, hijo de Josías, también llamado Joaquín, encontrado en 2 Re 23,34 (cf. 2 Cr 36,5-8). En Mt 1,11-12, este rey aparece con el nombre de Jeconías (cf. el final de la nota 23 de este artículo).

Azor es oriundo de la misma raíz del nombre Azarías, referente al rey de Judá, también llamado Ozías (cf. 1 Cr 3,12; 2 Cr 26; 2 Re 14,21$22 ; 15,1-7)$, el cual está en Mt 1,8-9. ${ }^{26}$

${ }^{24}$ Cf. Jeremias, Jerusalem, 294; y Brown, Messiah, 93.

25 Según DAVIES - AluISON, Matthew, 180, Mateo debe haber adoptado la LXX, porque ésta coloca a Salatiel como padre de Zorobabel en $1 \mathrm{Cr} 3,19$. En el mismo sentido, cf. D. J. HARRISON, The Gospel of Matthew, Sacra Pagina, Liturgical Press Minnesota - 2007, 29.

${ }^{26}$ Azor pertenece a la misma raíz de los nombres Azarías (cf. $2 \mathrm{Cr} 29,12$ ), Azur (cf. Jr 28,1; Ez 11,1) y Azareel (cf. 1 Cr 12,6). 

$8,17)^{27}$

Sadoc remite al sacerdote de David, en 1 Cr 6,8 y 18,16 (cf. 2 Sam

Ajim es una abreviación de Aquimaas, que se refiere al hijo del sacerdote Sadoc, aliado de David, como vemos en $1 \mathrm{Cr} 6,8-9$ y 6,53 (cf. 2 Sam 17,17). Entonces, no es por casualidad que en Mt 1,14 Ajim es descendiente de un tal Sadoc.

Eliud no se repite en la Biblia. Sin embargo, parece que este nombre está relacionado con Abiud (cf. Mt 1,13), ya que ambos se aproximan por los significados: Abiud, "Padre de alabanza", y Eliud, "Dios es mi alabanza". Además, excluyéndose a Jesús, ellos encabezan dos subgrupos de cinco nombres: de Abiud a Ajim y de Eliud a José. ${ }^{28}$

Eleazar recuerda al hijo de Aarón y antecesor de Sadoc, en $1 \mathrm{Cr} 6,3$ (cf. Nm 20,24-28 y Dt 10,6).

Entre los siete nombres arriba citados, es importante percibir que Eliaquim surge en Mt 1,11-12 en la forma de Jeconías, y Azor o Azarías en la forma de Ozías en Mt 1,8-9. También se debe observar la relación que existe entre Abiud y José (cf. Mt 1,16), Sadoc y Ajim, Eliud y Abiud, Eleazar y Sadoc (cf. Mt 1,14). Es interesante notar que los nombres Azarías, Sadoc y Aquimaas aparecen juntos en $1 \mathrm{Cr}$ 6,8-10. Tal vez este pasaje haya inspirado al evangelista para componer el conjunto AzorSadoc-Ajim de Mt 1,14.

Finalmente, agregamos que el nombre Matán (cf. Mt 1,15) puede estar abreviando el nombre Matanías, el cual designa al último rey de Judá antes del exilio babilónico, también llamado Sedecías, hijo de Josías y hermano de Eliaquim o Joaquín, constante en 2 Re 24,17 y 2 Cr 36,1 1 (en este pasaje Sedecías fue confundido con el hermano de Jeconías, hijo de Eliaquim). ${ }^{29}$

27 En este artículo se adoptó la numeración de la Vulgata para los versículos de los capítulos 5 y 6 del $1 \mathrm{Cr}$. En el Texto Masorético y en la LXX, 1 Cr 6,1-15 corresponde a 5,28-41, y $1 \mathrm{Cr}$ 6,16-81 a 6,1-66.

28 DAVIES - AlLISON, Matthew, 181, observan que el nombre Eliud aparece en el texto de la LXX del Códice Alejandrino, en $1 \mathrm{Cr}$ 12,20-21. Pero probablemente se trata de un error del copista, porque en los demás códices de la LXX está Elimuth y, en el Texto Masorético, Eliú.

${ }^{29}$ Matán también es el nombre de un sacerdote de Baal (cf. 2 Re 11,18; $2 \mathrm{Cr}$ 23,17). K. H. ReNGSTORF, Das Evangelium nach Lukas, NTD 3, Göttingen - 1965, 60, supone que el nombre Matán tiene que ver con el nombre Matat, que está en Lc 3,24, el cual, como Matán, antecede al abuelo de Jesús. JeREMIAS, Jerusalem, 292, afirma que no se puede tener seguridad de esto, porque el Matat de Lucas puede no ser 
Esta última observación parece ser significativa para entender la lista de Mt 1,13-15: a semejanza de lo que ocurrió en la historia de Judá, Matán o Matanías es el último miembro de la lista intermedia, preparándola para Jacob y José (cf. Mt 1,15-16), nombres que también remiten a otro exilio hebreo, que fue el de Egipto (cf. Gn 25,26; 30,22-24; Ex 1,114; $1 \mathrm{Cr}$ 2,1-2). Además, esta relación intencional analógica queda confirmada en Mt 2,13-15 (el refugio de José y Jesús en Egipto).

Frente a todas estas observaciones, podemos sugerir la hipótesis de que los nueve nombres constantes en Mt 1,13-16, desde Abiud hasta Jacob, poseen un fondo histórico-teológico que remite a los linajes sacerdotal, benjamita y davídico. Parece que este fondo determina una analogía tipológica entre el exilio de los judíos y el de Jesús en Egipto, con los nombres de Matán (Matanías), Jacob y José. ${ }^{30}$

Es posible que algunos nombres hayan sido abreviados (AzaríasAzor, Aquimaas-Ajim, Matanías-Matán) y repetidos por medio de modificaciones (Joaquín-Jeconías-Eliaquim, Ozías-Azarías-Azor y AbiudEliud), con la simple finalidad de rellenar los espacios y completar el número perfecto de catorce nombres en el tercer grupo. La posibilidad de esta técnica se confirma con las identificaciones que existen entre algunos nombres y especialmente con la función interna tipológica de Matán.

\subsection{La genealogía de Lc 3,23-38}

En el texto que analizaremos ahora no encontramos una declaración de los objetivos del evangelista para presentar su genealogía. Sin embargo, podemos afirmar, en líneas generales, que existe el propósito de indicar el linaje davídico, como también el origen o naturaleza humana y divina de Jesús, debido a la mención de los nombres de David, Adán y Dios, pero especializándolo a la raza hebrea por el nombre de Abraham. ${ }^{31}$

original. A decir verdad, considerándose la formación de todo el conjunto genealógico de Mateo, la tradición que éste adoptó es visiblemente independiente de la de Lucas. Por lo tanto, tal hipótesis no tiene razón de ser.

${ }^{30}$ La función tipológica del grupo Jacob-José-Jesús ya había sido observada por $\mathrm{H}$. C. WAETJEN, "The Genealogy as the Key to the Gospel According to Matthew", JBL 95 (1976) 205-230, esp. 225-227.

${ }^{31}$ La colocación de Dios en la lista de Lucas tiene la obvia intención de afirmar que Jesús desciende directamente de Dios y que, por eso, era de naturaleza divina. Ninguna otra lista genealógica del Antiguo Testamento y de textos no canónicos menciona a Dios. El texto veterotestamentario, de manera general, presenta a Dios 
La lista, formada por setenta y siete nombres principales, puede ser dividida en tres grupos, siendo que el primero contiene siete nombres y los demás son compuestos por treinta y cinco nombres cada uno, subdivididos en cinco grupos de siete nombres, totalizando setenta nombres. Al final de la lista, el nombre de Dios aparece como un excedente culminante. Veamos, entonces, cómo están dispuestos, separándolos por grupos y subgrupos, respetando al máximo la forma original griega en la transposición para el castellano: ${ }^{32}$

Grupo 1: Jesús, José, Elí, Matat, Leví, Melquí, Janái. Grupo 2:

2.1. José, Matatías, Amós, Nahúm, Eslí, Nangái, Maaz;

2.2. Matatías, Semeín, Josec, Jodá, Joanán, Resá, Zorobabel;

2.3. Salatiel, Nerí, Melquí, Addí, Cosán, Elmadán, Er;

2.4. Jesús, Eliezer, Jorim, Matat, Leví, Simeón, Judá;

2.5. José, Jonán, Eliaquim, Meleá, Menná, Matatá, Natán. Grupo 3:

3.1. David, Jesé, Jobed, Booz, Salá, Naasón, Aminadab;

3.2. Admín, Arní, Esrom, Fares, Judá, Jacob, Isaac;

3.3. Abraham, Tara, Najor, Serug, Ragáu, Fálec, Eber;

3.4. Salá, Cainán, Arfaxad, Sem, Noé, Lámec, Matusalén;

3.5. Henoc, Járet, Maleleel, Cainán, Enós, Set, Adán.

Excedente: Dios.

El principio formador de la lista en Lc 3,23-38 es el número siete, el cual se repite once veces para totalizar setenta y siete (sin contar a Dios al final). ${ }^{33}$ En la Biblia, el número siete indica la plenitud y, de hecho, en

meramente como creador. Por lo tanto, para Lucas, Jesús no era solamente hijo del hombre, por medio de Adán, sino también, era hijo de Dios, y el único que podría tener a Dios como su ancestro genealógico. Para esta cuestión, cf. R. T. Hood, "The Genealogies of Jesus", Early Christians Origins, FS H. R. Willoughby (ed. A. Wikgren), Quadrangle - Chicago - 1961, 14-15; FITZMYER, Luke, 504.

32 La lista de nombres de Lc 3,23-38 presentada en este artículo sigue la edición crítica de Nestle-Aland ${ }^{27}$.

33 Con respecto a la exclusión de Dios del recuento de los nombres, ver J. M. HeER, "Die Stammbäume Jesu nach Matthäus und Lukas", BS 15 1-2, Herder - Freiburg 1910. Como se verá a continuación, el sistema simbólico-numérico de Lucas exige la separación de Dios. Por otro lado, en la lista de Lucas el aislamiento de Dios parece ser 
los evangelios, la venida de Jesús significa la plenitud de los tiempos (cf. Mc 1,15; Mt 4,17; Lc 4,21; Jn 1,16), o sea, la época en que las profecías y las promesas divinas se cumplieron. ${ }^{34} \mathrm{Y}$ parece que Lucas desea mostrar cronológica e históricamente esta condición de plenitud en el número setenta y siete de su genealogía. ${ }^{35}$

El grupo 1 se destaca de los demás por tres razones:

a) él mismo parece constituir un principio formador, en especial para el segundo grupo, considerándose que los nombres Jesús y José surgen como cabezas en los subgrupos 2.1, 2.4 y 2.5, y los nombres Melquí, Matat y Leví se repiten en los subgrupos 2.3 y 2.4;

b) hay nombres que poseen formas semejantes, tanto en el primero como en el segundo grupo: José-Josec, Elí-Eslí-Elmadán-EliezerEliaquim, Matat-Matatías-Maaz-Matatá, Melquí-Meleá y JanáiJonán; y

c) su separación permite la ubicación de David entre el segundo y el tercer grupo, dentro del total simbólico de setenta nombres, el cual sirve como marca divisoria entre sus descendientes, que llevan directo a Jesús, y sus ascendientes, que se dirigen hacia Dios.

De los nombres del grupo 1, en la Biblia existen Jesús, José, Elí, Leví y Janái (cf. 1 Cr 5,12). En cuanto a Matat, se trata de una abrevia-

necesario, teniendo en cuenta que Dios trasciende el género humano y porque solamente Jesús puede ser Su descendiente legítimo.

${ }^{34}$ El significado de plenitud para el número siete fue atribuido inicialmente por Agustín, en De Gen. ad Litt. 4.16 y De Civitate Dei 11.31. El evangelio de Lucas parece insinuar este significado en más de dos pasajes: 8,2 (los siete demonios indicarían pleno dominio del Mal) y 10,1.17 (los setenta o setenta y dos discípulos denotarían no solamente numerosidad sino también plena acción y pleno poder del reino de Dios, como vemos en los vv. 18-20 del mismo capítulo).

35 Hay escritos no canónicos que dividen la historia del mundo en diez semanas de siete generaciones cada una, totalizando el número setenta, como 1 Henoc 93,1-10 (200 a. C.-50 d. C.). Otros dividen en doce períodos, como 2 Baruc 53-72 (110-120 d. C.), 4 Esdras 14,11 (70-120 d. C.) y Apocalipsis de Abraham 29 (70-150 d. C.). Existen estudiosos que presuponen la adopción de este último esquema por Lucas, mezclado con el de 1 Henoc. Así, el evangelista estaría relacionando once períodos de siete, siendo que el décimo segundo período vendría con la Era Mesiánica (cf. JEREMIAS, Jerusalem, 292). Pero tal vez lo más acertado sería pensar que Lucas y Mateo solamente deseaban formar una cantidad simbólica básica, o sea, de setenta y cuarenta, para después acrecentar y destacar grupos especiales en cada genealogía: para Lucas los primeros siete nombres (Jesús, José, Elí, Matat, Leví, Melquí y Janái, siguiendo su principio particular del siete) y para Mateo los dos últimos nombres (José y Jesús, que son los personajes principales de sus dos primeros capítulos). 
ción de Matatías. Y Melquí es una abreviación de varios nombres bíblicos, como se verá más abajo.

El grupo 2 es peculiar porque posee ciertos nombres desconocidos en la Biblia. Sin embargo, entre estos, algunos se aproximan a los siguientes nombres:

a) Josec: José (hijo de Jacob: Gn 30,24; la correspondencia simbólico-numérica establecida por Lucas entre estos dos nombres confirma la proximidad, como se verá más adelante);

b) Jodá: Judá (hijo de Jacob: Gn 29,35)

c) Resá: Rezón (adversario de Salomón: 1 Re 11,23-25);

d) Nerí: Ner (padre de Abner: 1 Sam 14,50-51; 1 Cr 9,39) y Nerías (padre de Baruc: Jr 32,12; 36,14);

e) Melquí: Melquiel (1 Cr 7,31; Nm 26,45; Gn 46,17; también puede ser transliterado Malquiel), Melquiram (1 Cr 3,18; también Malquiram), Melquisúa (1 Cr 9,39; también Malquisúa), Melquisedec (Gn 14,18-20), Melquías (1 Cr 6,40; también Malquías);

f) Addí: Adiel (1 Cr 4,36);

g) Elmadán (que también puede ser transliterado Elmodam): Elmodad (Gn 10,26; 1 Cr 1,20; también transliterado Almodad);

h) Jorim: Joram (2 Cr 21; 2 Re 1,17);

i) Jonán: Joanán (1 Cr 6,9-10; la correspondencia simbólico-numérica establecida por el evangelista entre estos dos nombres confirma la proximidad, como se verá más adelante).

Solamente Eslí, Nangái, Cosán, Meleá y Menná no poseen formas cercanas en la Biblia. ${ }^{36}$

A título de referencia y ubicación, mencionaremos también los nombres conocidos en la Biblia que están en el grupo 2:

a) José: hijo de Jacob (Gn 30,24);

b) Matatías: un sacerdote macabeo (en la LXX: 1 Mac 2,1), un levita (1 Cr 9,31) y un cantor (1 Cr 25,3);

${ }^{36}$ Sin embargo, Eslí posee cierta semejanza con Elí (que puede ser confirmada por la relación simbólico-numérica del evangelista, pues de Elí y Eslí hay diez nombres). También el nombre Nangái tiene proximidad de significado con Nerí: el primero significa "claridad", "brillo" y "luz", y, el segundo, "mi luz". Tal vez esta correspondencia pueda ser confirmada con el simbolismo numérico de Lucas, una vez que de Nangái a Nerí se cuentan diez nombres. 
c) Amós: el profeta (Am 1,1; en la LXX: Tb 2,6);

d) Nahúm: el profeta (Na 1,1; en la LXX: Tb 14,4);

e) Maaz: un levita (1 Cr 6,20; 2 Cr 29,12; 31,13);

f) Semeín, en la forma Semei: varios personajes; un hijo de Pedaías y hermano de Zorobabel (1 Cr 3,19);

g) Joanán: un guerrero de David (2 Re 25,23), el hijo de Josías (1 Cr 3,15), el hijo de Elioenai (1 Cr 3,24) y un sacerdote (1 Cr 6,9-10);

h) Zorobabel: el gobernador, descendiente de David (1 Cr 3,19; Esd 2,2; $\mathrm{Ne} 7,7)$

i) Salatiel (también Sealtiel): padre de Zorobabel (1 Cr 3,17; Esd $3,2)$;

j) Er: hijo de Judá (Gn 46,12; 1 Cr 2,3);

k) Jesús: el hijo de Sirac (en la LXX: Sir 51,30 o 18);

1) Eliezer: varios personajes; hijo de Moisés (Ex 18,4; 1 Cr 23,15.17);

m) Leví: hijo de Jacob (Gn 29,34);

n) Simeón: hijo de Jacob (Gn 29,33);

o) Judá: hijo de Jacob (Gn 29,35);

p) Eliaquim: rey, hijo de Josías (2 Re 19,2; 23,34);

q) Matatá: Esd 10,33;

r) Natán: el profeta (2 Sam 12,1; 1 Re 1,8), un hijo de David (1 Cr 3,$5 ; 2$ Sam 5,14).

Otro detalle de los grupos 1 y 2 es que en ellos existen subgrupos secundarios, además de los subgrupos sucesivos, los cuales se identifican por las letras iniciales, aunque con inversiones, desplazamientos y posiblemente repetición, modificación y añadidura de un nombre. Ellos se destacan en el momento en que formamos un nuevo grupo de cuarenta nombres, al juntar el grupo 1 con el grupo 2, pero excluyéndose los nombres de Zorobabel y Salatiel, que quedan en el centro de esta nueva lista. Estos cuarenta nombres componen el grupo donde están los ascendientes desconocidos de Jesús. A los efectos de comparar, dividiremos este grupo de cuarenta nombres en dos partes. La lista es la siguiente:

a) primera parte: Jesús, José, Elí, Matat, Leví, Melquí, Janái, José, Matatías, Amós, Nahúm, Eslí, Nangái, Maaz, Matatías, Semeín, Josec, Jodá, Joanán, Resá;

b) centro: Zorobabel y Salatiel; 
c) segunda parte: Nerí, Melquí, Addí, Cosán, Elmadán, Er, Jesús, Eliezer, Jorim, Matat, Leví, Simeón, Judá, José, Jonán, Eliaquim, Meleá, Menná, Matatá, Natán.

Vemos, entonces, dentro de las dos partes de este grupo, cuáles son los subgrupos secundarios:

a) J-J-E-M-L corresponde a J-E-J-M-L: Jesús-José-Elí-Matat-Leví corresponde a Jesús-Eliezer-Jorim-Matat-Leví (con inversión de José-Elí en Eliezer-Jorim);

b) S-J-J-J corresponde a S-J-J-J: Semeín-Josec-Jodá-Joanán corresponde a Simeón-Judá-José-Jonán (con inversión de Josec-Jodá en Judá-José);

c) E-N-M-M corresponde a E-M-M-M-N: Eslí-Nangái-Maaz-Matatías corresponde a Eliaquim-Meleá-Menná-Matatá-Natán (con desplazamiento en Nangái-Natán; posible repetición de Meleá, pero modificándolo en Menná; añadidura de un nombre, o sea, Menná, probablemente para formar treinta y cinco nombres en el grupo 2; modificación de la forma Matatías-Matatá) ${ }^{37}$

d) M-A-N corresponde a N-M-A: Matatías-Amós-Nahúm corresponde a Nerí-Melquí-Addí (desplazamiento en Nahúm-Nerí).

Es interesante notar que los subgrupos J-J-E-M-L y S-J-J-J surgen separados en la primera parte del grupo de cuarenta nombres (en el comienzo y en el final) y juntos en la segunda parte (en el centro), pero con J-J-E-M-L en la forma J-E-J-M-L. El subgrupo E-N-M-M surge antes del subgrupo S-J-J-J en la primera parte, y, después de él en la segunda parte, en la forma E-M-M-M-N. En cuanto al subgrupo M-A-N, aparece después de J-J-E-M-L en la primera parte y antes de él en la segunda parte, en la forma N-M-A, pero con J-J-E-M-L en la forma J-EJ-M-L.

Otra cosa interesante entre los grupos 1 y 2 es la repetición de nombres después de un cierto intervalo numérico, contándose sucesivamente, pudiendo haber varias combinaciones, tanto en orden recto como en el inverso:

37 De acuerdo con A. SChLATTER, en Das Evangelium des Lukas, Stuttgart - 1931, 218, habría ocurrido una diptografía en Meleá-Menná. 
a) seis grupos de 7, en orden recto: de Jesús a Janái $=7$, el siguiente es José; de José a Matatías = 7, el siguiente es Matatías; de Matatías a Zorobabel $=7$, el siguiente es Salatiel; de Salatiel a $\mathrm{Er}=7$, el siguiente es Jesús; de Jesús a Judá $=7$, el siguiente es José; de José a Natán $=7$, el siguiente es David;

b) seis grupos de 7 , en orden inverso: de David a Jonán $=7$, el siguiente es José; de José a Eliezer $=7$, el siguiente es Jesús; de Jesús a Nerí $=7$, el siguiente es Salatiel; de Salatiel a Semeín $=7$, el siguiente es Matatías; de Matatías a Matatías = 7, el siguiente es José; de José a José $=7$, el siguiente es Jesús;

c) cuatro grupos de 7 × $4=28$, en orden recto: de Jesús a $\mathrm{Er}=28$, el siguiente es Jesús; de Matat a Jorim $=28$, el siguiente es Matat; de Leví a Matat $=28$, el siguiente es Leví; de José a Judá $=28$, el siguiente es José;

d) cuatro grupos de 7 x $4=28$, en orden inverso: de José a Matatías = 28, el siguiente es José; de Leví a Melquí $=28$, el siguiente es Leví; de Matat a Leví = 28, el siguiente es Matat; de Jesús a José = 28, el siguiente es Jesús;

e) dos grupos de 7, tanto en el orden recto como en el inverso: de José a José $=7$; de Matatías a Matatías $=7$;

f) 6 × 3 = 18: de Melquí a Nerí = 18, el siguiente es Melquí.

Con nombres parecidos:

a) 5 × 2 = 10: de José a Josec = 10; de Elí a Eslí = 10;

b) 6 × 3 = 18: de Jodá a Judá $=18$; de Semeín a Leví $=18$, el siguiente es Simeón; de Joanán a José $=18$, el siguiente es Jonán;

c) 7 x 4 =28: Elí a Eliezer $=28$.

De estas posibilidades numéricas percibimos que despuntan algunos nombres-clave, es decir, los más repetidos: José, Matatías, Jesús, Leví y Matat. ${ }^{38}$

Tal vez podamos decir que la colocación numérica destacada de nombres-clave repetidos debe haberse basado en sus respectivos significados: José, "él añade", Matatías, "regalo de Dios"; Jesús, "Dios salva";

38 J. E. BRUns, "Matthew's Genealogy of Jesus", The Bible Today 1 (1964) 980985, ya había observado la existencia de nombres significativos dentro del intervalo de septenas, como José, Salatiel y Jesús. 
Leví, "ligado"; Matat, "regalo". Sin embargo, algunos otros repetidos, a veces modificados, también tienen significados importantes: Melquí, "mi rey”; Elí, “ofrenda”; y Judá, “alabanza del Señor”. Todos ellos remiten a la idea de sacerdocio y gobierno mesiánico, así como al hecho de que Jesús es un "regalo de Dios" (Matatías), por medio de José ("aquel que añade").

De todo este análisis concluimos que los grupos 1 y 2 están sistematizados de la siguiente manera:

a) una parte de los nombres que se suceden después del grupo 1, de José hasta Natán, siguen el patrón de los nombres que componen el grupo 1;

b) éstos también se aproximan a nombres que constan en el Antiguo Testamento, habiendo, en parte, nombres idénticos $\mathrm{y}$, en parte, formas abreviadas y un poco modificadas;

c) la ubicación de los nombres respeta una organización por medio de algunos subgrupos secundarios de letras iniciales, que fueron puestos distanciados o próximos, de forma alternada, conforme dos mitades de un grupo de cuarenta nombres, separados por Zorobabel y Salatiel, que están en el centro de este grupo; y

d) la ordenación también siguió un sistema de nombres-clave que se repiten después de una secuencia numérica de siete, veintiocho, dieciocho y diez, abarcando algunos nombres semejantes en la forma.

Debemos resaltar otro detalle importante en la formación de los grupos 1 y 2. Este detalle es el desvío hacia Natán. Sin duda, este desvío determina que Jesús no sea descendiente de ninguno de los reyes que reinaron después de David. Tal vez esta determinación indique una intención de diferenciar el reino de Jesús de algo esencialmente político y humano; su reino es puramente espiritual y no participa de los pecados históricos y notorios de la monarquía israelita. Por otro lado, el desvío hacia Natán puede haber sido el resultado de la lectura de Za 12,12.

Con referencia al grupo 3, se puede percibir que fue copiado de algún texto defectuoso de $1 \mathrm{Cr}$, que tuvo como fuente a la Septuaginta, considerando los errores en la escritura de los nombres y la colocación repetida de Cainán (la Septuaginta repite este nombre entre Arfaxad y 
Salá en 1 Cr 1,24). ${ }^{39}$ Parece que este texto defectuoso fue conveniente para el evangelista, especialmente para hacer valer sus principios numéricos de septenas y de intervalos, ya que, en el orden inverso, la repetición de Cainán después de ocho nombres propicia que sean completados treinta y cinco nombres hasta David, así como permite continuar grupos de siete con importantes nombres a la cabecera, como Henoc y Abraham.

También dentro del intervalo de septenas, en orden inverso, surgen como nombres principales: Henoc, Salá, Abraham y Admín. El comienzo y el final de la lista son señalizados por Dios (un excedente) y David, mientras en el medio se encuentra Abraham (detalle que también es suscitado por la repetición de Cainán).

En el grupo 3, llaman la atención los nombres de Admín y Arní.

Se acostumbra decir que el primer nombre es una abreviación de Aminadab. ${ }^{40}$ Pero parece que él tiene más que ver con una formación hecha a partir de la misma raíz de Admá (Gn 10,19; Dt 29,23), Adumín (Jos 15,7) y Adán (Gn 3,20). ${ }^{41}$ Una configuración simbólico-numérica que puede confirmar la relación de raíz entre estos nombres es el hecho de que de Admín a Adán existen veintiocho nombres (o sea, 7 x 4). Por otro lado, debido al hecho de que Admín no aparece en $1 \mathrm{Cr} 2,10$, tal vez él sea una simple añadidura para permitir que en los grupos 3.3 y 3.5 surjan los nombres-cabecera Abraham y Henoc, como hacen MeleáMenná (en el grupo 2) y Cainán-Cainán.

En cuanto a Arní, parece un error cometido por parte del copista, porque en su lugar debería estar Aram o Ram. Pero se acerca bastante a la forma del nombre Arnán (cf. 1 Cr 3,21). El copista puede haberse

39 De acuerdo a la sugerencia de G. KUHN, "Die Geschlechtsregister Jesu bei Lukas und Matthäus nach ihrer Herkunft untersucht", ZNW 22 (1923) 216, el grupo 3 concuerda en general con la morfología de la LXX.

40 De acuerdo con la sugerencia de KUHN, "Geschlechtsregister", 217, n. 2. A causa de esta suposición, Aminadab no podría estar en la lista primitiva de Lucas (en B y en sy. ${ }^{\text {sin }}$ falta ese nombre). Sin embargo, su presencia es necesaria debido a su aparición en $1 \mathrm{Cr}$ 2,10 y en los demás códices, así como al acabado simbólico-numérico del grupo 3.

${ }^{41}$ Algunos manuscritos del Nuevo Testamento, como $\mathrm{P}^{4}, 01 *, 1241 \mathrm{y} \mathrm{sy.}{ }^{\text {sin }}$, tal vez confirman esta tesis, teniendo en cuenta que aparece en ellos el nombre Adán, ora en lugar de Aminadab, ora en lugar de Admín. 
confundido entre 'Aram y 'Arnan, y, por un error de lectura, de escritura, de pronunciación o de escucha, acabó escribiendo 'Arní. ${ }^{42}$

Una particularidad simbólico-numérica del grupo 3 son los nombres de Salá y nuevamente Cainán, que se repiten en una sucesión de $18(6 \times 3)$ y $10(5 \times 2)$ nombres, respectivamente, como ocurre con algunos ancestros del grupo 2 .

Al analizar las posibilidades numéricas entre los tres grupos, también encontramos algunas peculiaridades interesantes: ${ }^{43}$

a) 6 × 3 = 18: de Jesús (grupo 1) a Jodá (grupo 2) = 18; de Jodá a Judá (ambos del grupo 2) = 18; entre Judá (grupo 2) y Judá (grupo 3) $=18$ nombres intermediarios;

b) si concebimos un círculo que comienza con Jesús y termina con Adán, podemos encontrar lo siguiente: 26 nombres de Addí a Admín; 52 nombres de Addí a Set, o sea, 26 x 2, siendo que el siguiente es Adán; entre Admín y Adán existen 26 nombres intermediarios; y de Adán a Addí (repasándose para la lista encabezada por Jesús, del grupo 1 en adelante) tenemos también 26 nombres. Esta peculiaridad debe confirmar una vez más la relación por la raíz entre Admín y Adán, y, en añadidura, por semejanza, entre Addí y Adán.

Hay estudiosos que defienden la idea de que Lucas es más auténtico que Mateo. ${ }^{44}$ Pero debe decirse que es difícil aceptarla definitiva y cabalmente, en vista de la construcción artificial por medio de números, colocación de nombres repetidos en intervalos numéricos, abreviaciones nominales, subgrupos de iniciales, nombres desconocidos y objetivos

42 KUHN, "Geschlechtsregister", 217, n. 3, sugiere que Arní debe ser producto de una manera de leer el hebraico Aram. Tal vez haya habido una corrupción de la forma griega 'Aram a 'Arní. También se puede pensar que Arní venga de 'Arran, encontrado en el texto de Rut 4,19 LXX (cf. J. Nolland, Luke 1-9:20, WBC 35A, Word Books Dallas - 1989, 172).

${ }^{43}$ Debemos recordar que Brown, Messiah, 91, observa que Agustín y los Padres de la Capadocia ya habían encontrado un patrón numérico en la lista genealógica de Lucas. Brown también añade que en ella existen siete patriarcas de Adán hasta Henoc y setenta nombres entre Henoc y Jesús, así como veintiún nombres en el período del post-exilio, veintiún nombres en el período monárquico, catorce nombres en el período premonárquico de David hasta Isaac y veintiún nombres de Abraham hasta Adán, formando el siguiente patrón numérico: $21(3 \times 7)+21+14(2 \times 7)+21$.

44 Por ejemplo, JeREMIAs, Jerusalem, 297, piensa que la genealogía de Lucas es más auténtica que la de Mateo. 
teológicos, especialmente el de determinar que Jesús no era descendiente de los reyes judíos después de David. Toda esta técnica indica que la lista es una elaboración, o, por lo menos, que Lucas puede haber utilizado una lista primitiva, pero editándola y ampliándola sistemáticamente. ${ }^{45}$ Lo mismo se debe decir de Mateo, con la diferencia de que éste buscó hacer una reducción, no una ampliación. ${ }^{46}$

\section{Conclusión}

En el presente artículo quedó bien demostrado que las genealogías de Jesucristo fueron elaboradas conforme a modelos de nombres y linajes, siguiendo la cronología y la historia bíblica, y de acuerdo a prin-

45 La presencia de nombres sin forma bíblica previa o próxima, como Nangái, Cosán, Meleá y Menná y la frase hōs enomízeto ("como se pensaba") de Lc 3,23, parecen indicar esta posibilidad, a pesar de que en el caso de la frase se haya preferido decir que ella significa que "se pensaba" que José y sus ascendientes eran el padre y los ancestros de Jesús, cuando, en realidad, no lo eran, teniendo en cuenta el origen divino de este último. Pero la frase podría estar induciendo a la idea de que el evangelista copió la lista a partir de otro documento, o reprodujo una lista de determinada tradición oral, o que ella es resultado de una investigación propia (cf. Lc 1,3). Asimismo, es visible la preocupación artificial y teológica de la lista, lo que induce a la idea de que hubo una elaboración o manipulación y ampliación, algo que no es imposible, teniendo en cuenta que en muchos puntos del evangelio de Lucas hay indicios de que este evangelista utilizó su creatividad, como enseña W. G. KüMMEL, en Einleitung in das Neue Testament, Quelle \& Meyer - Heidelberg - 1973, 110-111, cuando comenta, por ejemplo, la "narrativa del viaje" (LC 9,51-19,27), composición de exclusiva autoría lucana elaborada por el evangelista para llenar el vacío creado por Mc 10,$1 ; 11$, 1. Para la cuestión, véase también FITZMYER, Luke, 499-500.

${ }^{46}$ En $1 \mathrm{Cr}$ existen versiones de genealogías, en las cuales se añaden o se suprimen nombres (cf. $1 \mathrm{Cr}$ 2,3-3,4 y 4,1-23; 7,6-12 y 8,1-40). Los motivos de estas contradicciones deben basarse en la necesidad de legitimar las filiaciones y en el hecho de que el cronista decidió conscientemente dejar registradas todas las posibilidades de genealogías, según las varias tradiciones que recogió. Ya en el caso de Lc 3,23-38, parece existir la intención sistemática de presentar una cronología que conforme una lista de número perfecto (setenta y siete). Naturalmente, a causa del desvío hacia Natán, los nombres entre éste y Salatiel no podrían coincidir con los que son proporcionados por Mateo, ya que él optó por los descendientes de Salomón. Es por eso que existe la diferencia de nombres. Pero tanto Mateo como Lucas no aceptaron 1 $\mathrm{Cr}$ 3,19-20, prefiriendo extraer y modificar nombres. Una cosa es cierta: por motivos teológicos, Salatiel y Zorobabel tenían que constar en las genealogías, fuesen o no fuesen descendientes regios (especialmente porque Zorobabel posee la condición mesiánica en Ag 2,21-23 y Za 4,6-10). Sólo que Lucas, al contrario de Mateo, calculó con mayor precisión el tiempo y la cantidad de generaciones necesarias para llegar hasta esos personajes, porque no se ató a un principio numérico muy limitado, como hizo Mateo. 
cipios internos simbólico-teológicos, que obedecieron a intervalos de grupos numéricos significativos.

Fueron puestos en evidencia aquí algunos detalles nuevos, entre los cuales los más importantes son los siguientes:

1. En cuanto a las tesis de armonización y de inclusión de María, la alternativa hipotética de que ésta podría ser descendiente de David por medio de otro hijo, aparte de Salomón y Natán, la cual nos ayuda a descartar aquellas tesis complicadas.

2. En el caso de la genealogía de Mateo, el aprovechamiento de otros nombres diferentes atribuidos a algunos ancestros, como Eliaquim, referido a "Joaquín-Jeconías", y Azor, referido a Ozías; la abreviación de Aquimaas para Ajim y de Matanías para Matán; la posible modificación de Azarías para Azor, teniendo en cuenta 1 Cr 6,8-10, cuyo pasaje debe haber inspirado la creación del conjunto Azor-Sadoc-Ajim; las relaciones entre Abiud y José, Sadoc y Ajim, Eliud y Abiud, Eleazar y Sadoc; y la función introductoria y tipológica de Matán, en el grupo Matán-Jacob-JoséJesús.

3. Con respecto a la lista genealógica de Lucas, la constatación de que el grupo de los primeros siete nombres sirve como principio generador de otros nombres, que los destaca del resto de la genealogía; la posible influencia de algunas formas nominales encontradas en el Antiguo Testamento; el reaprovechamiento de nombres, pero con la modificación de las formas, como José-Josec, Elí-Eslí, Judá-Jodá, Semeín-Simeón y Joanán-Jonán; la existencia de subgrupos organizados de acuerdo con las letras iniciales de los nombres; la relación simbólico-numérica entre nombres con formas próximas; la constante numérica entre los intervalos de siete, veintiocho, dieciocho y diez, así como, subsidiariamente, de veintiséis y cincuenta y dos; la posible utilización de significados para la elección de nombres-clave; la intención teológica de evitar dar a Jesús una condición regia israelita, mediante el desvío hacia Natán; la relación por semejanza y por la raíz de los nombres Addí, Admín y Adán, confirmada por el sistema simbólico-numérico del evangelista; y la propuesta de que Arní es más próximo de la forma Arnán.

En el caso de Lucas, es importante añadir su preocupación por repetir nombres que, de acuerdo con sus significados originales, apuntan 
hacia Jesús. Por este motivo, podemos decir que su lista refleja un cristocentrismo. Pero la manera en que la dispone, parece insinuar algo semejante a lo que afirma Ap 1,8.17; 22,13: Jesús y Dios son el principio y el fin de toda la Historia. De lo que se desprende que la lista de Lucas posee una amplitud teológica mayor que la de Mateo.

ADYLSON VALDEZ

SANTOS, BRASIL

adylv@lbm.com.br 